\title{
LOS TRABAJADORES AGRÍCOLAS MEXICANOS EN LOS CAMPOS DE CALIFORNIA: MIGRACION, EMPLEO Y FORMACIÓN DE CLASE EN UNA AGRICULTURA INTENSIVA.
}

Mexican farmworkers in California's fields: migration, employment and class formation in an intensive agriculture.

\section{MANUEL ADRIÁN HERNÁNDEZ ROMERO*}

Fecha de recepción: 29 de julio de 2015- Fecha de aprobación: 7 de septiembre de 2015

\section{Resumen}

En este artículo se presenta una aproximación antropológica al proceso de formación de clase, motivado por el asentamiento y estabilización de la fuerza de trabajo migrante mexicana empleada en la agricultura de California. A partir de observación directa de las dinámicas productivas de una mercancía ilustrativa, las uvas para mesa, se describen las pautas de participación laboral en un contexto de capitalismo agrícola avanzado para explorar las formas de proletarización vinculadas al uso de mano de obra externa, analizando tanto los factores que propician proletarización como las condiciones que impiden que el empleo agrícola se iguale a otros sectores de la economía.

Palabras clave: trabajadores agrícolas, proletarización, migrantes mexicanos, agroindustria.

\section{Abstract}

This article presents an anthropological approach to the process of class formation driven by the settlement and stabilization of the Mexican migrant workforce employed by California's agriculture. Based on direct observation of productive dynamics in an illustrative commodity, that is table grapes, labor participation in advanced agrarian capitalism is described, in order to explore proletarianization forms related to use of external labor force, analyzing features leading to proletarianization as well as conditions preventing farm work of being equal to other sectors of economy

Keywords: farmworkers, proletarianization; Mexican migrants; agribusiness.

* Doctor en Antropología, CIESAS. Investigador independiente. Correo-e: maehr2001@yahoo.com.mx 


\section{Presentación}

En el paisaje intelectual de la antropología latinoamericana las investigaciones que abordan temas relacionados con la cuestión agraria ocupan un lugar relevante. En México, uno de los desarrollos más notorios fruto de ese interés fue el llamado debate entre campesinistas y descampesinistas, en el que se discutió, entre otras cosas, el destino del campesinado ante los embates de la expansión capitalista (para un recuento de la discusión ver Feder, 1977). Sin regresar al lugar central que tuvieron en esos días, varios de los temas y cuestionamientos planteados entonces resurgen periódicamente, ante la cambiante realidad de la sociedad mexicana. En este escrito recuperaremos uno de esos temas: la proletarización del trabajo agrícola. Sin embargo, lo haremos con un desplazamiento geográfico, para explorar lo que ha sido uno de los destinos del campesinado mexicano: la migración hacia los Estados Unidos, especialmente a California, para emplearse como jornaleros agrícolas.

La presencia masiva de trabajadores migrantes mexicanos es desde hace varias décadas uno de los componentes esenciales en el funcionamiento de la agricultura californiana (Palerm, 1991, 1999; Durand \& Massey, 2003: 147-163). Esto representa un escenario propicio para abordar el tema de la proletarización dentro de una forma de organización plenamente capitalista. A partir de una etnografía del espacio de trabajo, aquí proponemos una mirada antropológica al proceso de formación de clase. Con base en el estudio de la dinámica productiva de las uvas para mesa en el sur del Valle de San Joaquín, se describen las condiciones de estabilización laboral que algunos migrantes provenientes de México encuentran en el medio rural californiano, indagando sobre el tipo de trabajador que surge en este contexto. La información presentada proviene de dos estancias prolongadas de trabajo de campo antropológico, llevadas a cabo en el Valle de San Joaquín, en California, entre 2005 y 2007, como parte de una investigación de posgrado cuya tesis doctoral presenta más extensamente algunos de los temas aquí tratados (Hernández, 2010). La investigación incluyó entrevistas semi-estructuradas con actores involucrados en distintos roles dentro de la producción agrícola (productores y empresarios, supervisores, jornaleros, extensionistas, activistas y funcionarios laborales), sondeos y cuestionarios a grupos de trabajo, reconstrucción de trayectorias migratorias y laborales, además de privilegiar la observación directa y recurrente en los espacios productivos y el seguimiento continuado de los grupos de trabajo para documentar las experiencias de los trabajadores y comprender su dinámico ciclo de empleo.

El texto comienza con una breve introducción a la cuestión de la caracterización de los asalariados agrícolas en términos de clase. Luego se presenta el contexto del caso de estudio con el propósito de destacar la importancia de la uva de mesa y definirla como un ejemplo ilustrativo de orientaciones productivas más amplias. A continuación sigue una sección dedicada a la descripción de la dinámica productiva, en donde se intenta mostrar las necesidades laborales del sector. Luego de presentar el espacio de trabajo, se exponen ciertos factores relevantes en la estabilización de los trabajadores, especialmente su asentamiento en el espacio rural californiano. Finalmente, el texto cierra con una breve reflexión de corte teórico sobre lo que este proceso implica. 


\section{Planteamiento}

Las discusiones sobre la caracterización en términos de clase de la fuerza de trabajo vinculada al capitalismo agrícola cuentan con una larga tradición dentro de las ciencias sociales, con enigmas e interrogantes que han captado la atención de diversos estudiosos. El propio Marx, al analizar los procesos de acumulación y proletarización en el campo británico (Marx, 1975[1867]: 866-67), detectó una de las tensiones fundamentales al señalar las extremas fluctuaciones de las necesidades del insumo trabajo en la producción agrícola. Elaborando sobre ello, K. Kautsky (1974 [1899]: 153 y ss.) habló de los límites que la agricultura impone al capitalismo para repetir las condiciones de estabilización, regularidad y concentración en el uso del trabajo que se consiguieron en las fábricas y generaron el proletariado industrial, hallando funcional para la gran propiedad capitalista la coexistencia de otras modelos de agricultura (básicamente, pequeños productores campesinos) que subsisten en cuanto pueden dotarla, temporalmente, de fuerza de trabajo. Estas relaciones de coexistencia, ya entendidas teóricamente como articulación, resultaron posteriormente de gran interés para los antropólogos, quienes enfatizan en las condiciones de asimetría y desigualdad y en los mecanismos de transferencia de valor que favorecen la acumulación del capital (A. Palerm, 1998). En décadas recientes, algunas de estas interrogantes se replantean a la luz de la escala global de la integración de los sistemas de producción de alimentos, cuestionando la manera en que diversos tipos de producciones locales a lo largo del mundo se vinculan con grandes compañías transnacionales, cadenas de supermercados y circuitos migratorios (Bonnano,
Busch, Friedland, Gouveia \& Mingione, 1994).

En el caso de Estados Unidos, la caracterización de la mano de obra agrícola también ha resultado problemática. Aunque se trata de un claro ejemplo de economía capitalista, la figura del trabajador agrícola asalariado ocupa un lugar extraño y de cierta forma negado en el imaginario de su estructura de clases. Como apunta GuerinGonzalez (1994:14), en la versión americana del mito agrario, centrada en la figura de los pequeños propietarios, "el trabajo asalariado parecía ser incidental y extraordinario más que central a la producción agrícola. Los proletarios no tenían lugar en este mito". A menudo, el empleo agrícola se concebía como la última oportunidad para los incapaces de conseguir mejores opciones, como una alternativa pasajera en momentos de crisis o incluso como un recurso de subsistencia de algunos de los miembros menos virtuosos de la sociedad, como los indigentes o los alcohólicos (Fuller, 1960, 1974). Paulatinamente, la figura del inmigrante, especialmente originario de México e indocumentado, fue asociándose al trabajo agrícola, reforzando su percepción como algo marginal y extraño.

En este contexto, el caso de California ha representado una excepción especial, con un factor distintivo desde épocas tempranas (finales del siglo XIX): una agricultura pautada no por los pequeños productores sino por la gran propiedad, para la que laboran grandes masas de trabajadores asalariados, siempre de origen foráneo (McWilliams, 1969[1939]; Palerm, 1999)1. Aunque la sugerente imagen de "fábricas en los campos" que uso C. McWilliams para definir la agricultura californiana llamó la atención, esta numerosa presencia de asalariados no condujo necesariamente a la percepción de la formación de una clase trabajadora, imperando más bien la 
imagen de un sector caracterizado por el empleo casual, sin patrones de trayectoria ocupacional y con nula o muy reducida integración en las relaciones laborales o entre los propios ejecutantes de la labores (Fuller, 1960). En uno de los más influyentes estudios en la materia, Fisher caracterizó el empleo agrícola californiano como un "mercado de trabajo no-estructurado", que no estaba regido -en términos generalespor ningún principio o instancia para regular $u$ organizar el trabajo en torno a habilidades, relaciones contractuales formales, división y jerarquización de tareas o estratificación salarial, siendo un espacio que incorporaba y desechaba una mano de obra indiferenciada, anónima, no calificada y estacional, todo lo cual contribuía a que fuera altamente inestable (Fisher, 1953: 7-9). La desfavorable situación resultante para quienes ejecutaban el trabajo agrícola, se pensaba tendría su solución en una transformación que se imaginaba inevitable: la eliminación de la necesidad de trabajadores gracias a la mecanización de las tareas (Ídem: 148).

Una cadena de éxitos en la sustitución del trabajo humano por máquinas en diversas cosechas hacía pensar que efectivamente la incómoda presencia de los jornaleros agrícolas llegaría a su fin, dejando su lugar a un más reducido cuerpo de operarios de maquinaria, quizá más acordes con la imagen de una economía moderna. Sin embargo, el desarrollo del sector agrícola en California dio un giro, desviándose de este esperado destino: desde mediados de la década de 1970 la producción se volcó a ciertas mercancías intensivas en trabajo, expandiendo la demanda de trabajadores. Los migrantes mexicanos, siendo para entonces los principales empleados en los campos del estado, aumentaron su presencia considerablemente. Como apuntara J. Palerm (1999: 161), la agricultura industrial californiana "en lugar de mecanizarse, se mexicanizó". Es en este contexto de intensificación y mexicanización, vigente hasta la fecha (CRB, 2013), que este artículo se aproxima a las dinámicas productivas actuales para observar las formas de participación laboral que hoy existen en esta agricultura.

\section{EI valle de las uvas}

California es el estado de la Unión americana cuya producción agrícola alcanza el valor monetario más elevado, sobrepasando la marca anual de los 40 mil millones de dólares (CDFAs/f). Aunque extendida a lo largo del territorio estatal, la agroindustria alcanza semejante volumen gracias al sustancial aporte de ciertas regiones específicas, y dentro de ellas la más importante se encuentra en el extremo sur del Valle de San Joaquín, con los condados de Fresno, Kern y Tulare generando una tercera parte del valor total (Ídem). Al estudiar la composición productiva de estos condados puede apreciarse con mayor claridad la orientación tomada por el sector agrícola californiano, que hoy privilegia el cultivo de lo que se conoce como el núcleo de frutas, nueces y vegetales (FNVs), de mayor valor en el mercado, en sustitución de los denominados field crops (cereales, fibras y leguminosas) que por la mecanización y la competencia internacional se han depreciado. Durante las últimas cuatro décadas el incremento de la superficie dedicada al primero de esos grupos es notorio, aparejado a la disminución del segundo. Por ejemplo, mientras la superficie dedicada a los field crops en el condado de Kern representaba el $75 \%$ del terreno cultivado en 1975 , para 
2010 había disminuido al 45\%. En contraparte, durante el mismo periodo el conjunto de frutas, nueces y vegetales pasó de abarcar el 22 al 54\% (KCDA, 2015).

En esta tendencia, algunos productos de alto valor en el mercado comenzaron a cobrar una relevancia cada vez mayor, en detrimento de los que descendían en rentabilidad. Así, en el Condado de Kern, el algodón, alguna vez conocido como el "rey" de los campos, perdió paulatinamente la posición de liderazgo como la mercancía individual con mayor contribución al valor de la producción, disminuyendo su aporte de un $22 \%$ en 1975 a un modesto $3 \%$ en el 2010. Durante ese mismo periodo, las uvas para mesa se fueron consolidando como producto líder, cuadruplicando su superficie e incrementando su participación en el valor conjunto del condado, pasando de representar solamente un 3\% a alcanzar el 11\% (KCDA, 2015). El auge de la producción de uva para mesa -y el de las FNVs en general- responde tanto a una expansión de las exportaciones como a un aumento de la demanda en los mercados internos. A la par que se incrementan las ventas a México, la Unión Europea y el mercado asiático (los principales compradores internacionales), nuevos hábitos alimenticios y de consumo dentro de los mismos Estados Unidos, impulsados por las preocupaciones por una dieta sana y la promoción federal del consumo de frutas y vegetales frescos (Guthrie, Lin, Reed \& Stewart, 2005), generan un espacio creciente para la colocación del producto. Resultado de ello, el promedio anual de consumo de uvas para mesa pasó de tres a ocho libras per cápita entre 1970 y fechas recientes (Boriss, Brunke \& Kreith, 2006).
Con aproximadamente dos terceras partes de la superficie que en el estado se dedica al cultivo de uvas para mesa ubicadas en los condados de Kern y Tulare, el sur del Valle de San Joaquín se convierte en la región más importante para el producto, con la pequeña ciudad de Delano y sus alrededores como sede de casi todas las compañías del ramo. El cultivo de uvas de mesa comenzó en esta región a principios del siglo $X X$, cuando la actividad agrícola se expandía aceleradamente. Entre los primeros productores se encontraba un grupo de migrantes europeos provenientes de la Isla de Hvar, hoy parte de Croacia, en el Mar Adriático, quienes establecieron los viñedos e iniciaron el funcionamiento de algunas compañías que, ahora con operaciones de mayor tamaño y convertidas en corporaciones, siguen funcionando (Krissman, 1996: 98 y ss.). Algunos de estos primeros productores trajeron consigo las prácticas desarrolladas en el Viejo Mundo durante varios siglos de experiencia, pero una vez traídos a California estos conocimientos han sido enormemente potenciados por las instrucciones técnicas elaboradas por los Departamentos de Extensión Agrícola de la Universidad de California, que han desarrollado distintas variedades e introducido mejoras y nuevas prácticas basadas en investigaciones científicas de vanguardia, a partir de lo cual se ha conseguido una producción adaptada a las exigencias de los mercados actuales (Wood, McGuinnis \& Core, 2006).

Pese a las considerables implementaciones tecnológicas y de capital que han generado notables incrementos tanto en la calidad como en la cantidad de la producción, las uvas continúan siendo una mercancía intensiva en el empleo de mano de obra. Se han introducido nuevas 
variedades, diseñado sistemas de regadío más efectivos e incorporado avances para su manejo, conservación y transporte, pero los métodos manuales de cosecha y tratamiento de las plantas han cambiado poco y siguen demandando grandes contingentes de trabajadores ${ }^{2}$. Por ello, la expansión del cultivo ha contribuido al proceso por el cual se incrementa la presencia de fuerza de trabajo agrícola en la zona. En la trayectoria histórica, el sector ha empleado distintas oleadas migratorias que han nutrido los contingentes de trabajadores, pero desde mediados del siglo $\mathrm{XX}$ los migrantes mexicanos aumentaron paulatinamente su participación en este espacio laboral, hasta convertirse en la abrumadora mayoría durante las décadas recientes ${ }^{3}$.

\section{Los viñedos: su ciclo y espacio de trabajo}

Al igual que la mayoría de las mercancías agrícolas, las uvas para mesa tienen un ciclo productivo con requerimientos variables en cuanto al insumo de fuerza de trabajo, registrando tanto un periodo "pico" -durante la realización de la cosecha-con alta demanda de trabajadores, como lapsos de actividad prácticamente nula. Pero hay dos características dignas de mención que influyen de manera importante en la constitución de su base de trabajadores. Primero, a diferencia de otras mercancías en las que la temporada de cosecha dura tan solo unas cuantas semanas, aquí se extiende por varios meses, comprendiendo en esta zona un periodo que arranca en julio para terminar en noviembre. Y segundo, en la preparación de su producción se involucran varios procesos que, sin llegar a alcanzar los requerimientos laborales de la temporada alta, también movilizan trabajadores, generando un calendario de faenas que brindan una base de empleo que se extiende por casi el año entero.
El volumen total de trabajadores que forma el contingente de empleados de campo de una compañía se organiza regularmente con la constitución de equipos que reciben el nombre coloquial de cuadrillas. Para satisfacer la demanda variable que se desprende del ciclo, estas cuadrillas se expanden y contraen siguiendo los requerimientos mandados por la producción, incorporando y deteniendo a parte de sus miembros conforme cambian las actividades a realizar. Si tomamos a estas cuadrillas como unidad de referencia en una breve exposición del ciclo productivo podremos ver la dinámica de la participación de la fuerza de trabajo en el sector.

Durante la época de la cosecha las cuadrillas están integradas por entre 55 y 60 miembros, que trabajan bajo la dirección de dos "mayordomos" o capataces. La composición por género es prácticamente balanceada. En estos momentos, el trabajo del grupo consiste en cortar los racimos de uva, limpiarlos y darles forma si es necesario, y empacarlos inmediatamente, en el mismo campo, de donde serán llevados a una planta de enfriamiento y almacenaje. Para esta labor, la cuadrilla se organiza en equipos más pequeños, por lo general parejas, que conjuntan a un responsable de cortar el producto ("pizcador") y a otro encargado de embolsarlo y acomodarlo en cajas ("empacador"). Esta temporada tiene una duración aproximada de cinco meses, comenzando los primeros días de julio en la parte sur del Valle y avanzando hacia el norte para llegar a las cercanías de la ciudad de Fresno hacia finales de noviembre.

Tras un descanso de un par de semanas una vez que la cosecha concluye, las cuadrillas regresan a la actividad al acercarse el invierno, 
cuando los viñedos que se cosecharon en las semanas o meses anteriores son podados, para dejar únicamente el cuerpo de la planta con las ramas que servirán de base para la próxima producción. En ese estado, la viña "dormirá" durante las bajas temperaturas de entre diciembre y febrero. En este momento las cuadrillas tienen su composición más reducida, entre 20 y 25 miembros, con un marcado predominio de varones (total en algunos casos) y una preferencia por trabajadores que cuentan con experiencia o trayectoria en el grupo. Durante le época de poda, las cuadrillas ejecutan también otras tareas de cuidado, reparación y limpieza de viñedos, tendido o mantenimiento de sistemas de regadío y preparación de nuevos campos que se incorporan al cultivo, las cuales duran hasta finales de febrero.

Aquí sigue el receso continuo más largo, durante prácticamente todo el mes de marzo, periodo en el que las compañías descansan sus elementos de campo. Para comienzos de abril, los grupos de trabajo entran en acción nuevamente, dedicándose ahora a una nueva serie de tareas que tienen como fin garantizar que la próxima producción tenga la calidad y cantidad deseada. Algunas de estas tareas, como arrancar los brotes improductivos que crecen en las plantas, se consideran sencillas y no requieren adiestramiento, mientras que otras, como la de darle forma y tamaño a los nuevos racimos de uva conforme van creciendo, son más delicadas, se reservan para experimentados y demandan una mayor supervisión. Durante este periodo el tamaño de las cuadrillas aumenta paulatinamente, comenzando con alrededor de 30 elementos en abril para sumar 50 en junio. La presencia de mujeres crece también hasta llegar a una proporción balanceada con la de varones, tal como la tiene durante la época de cosecha, que comenzará nuevamente de manera inmediata estas tareas.

\begin{tabular}{|c|c|c|c|c|c|c|c|c|c|c|c|}
\hline \multicolumn{3}{|c|}{ Preparación } & \multicolumn{5}{|c|}{ Cosecha } & \multicolumn{3}{|c|}{ Poda } & Inactividad \\
\hline Abr. & May. & Jun. & Jul. & Ago. & Sept. & Oct. & Nov. & Dic. & Ene. & Feb. & Marzo \\
\hline \multicolumn{3}{|c|}{$\begin{array}{l}\text { Crecimiento paula- } \\
\text { tino de los grupos de } \\
\text { trabajo, comenzando } \\
\text { con } 30 \text { miembros } \\
\text { en abril y rondando } \\
\text { los } 50 \text { para junio. La } \\
\text { proporción de muje- } \\
\text { res aumenta gradual- } \\
\text { mente durante la } \\
\text { expansión del grupo. }\end{array}$} & \multicolumn{5}{|c|}{$\begin{array}{l}\text { Las cuadrillas se integran por } \\
\text { entre } 55 \text { y } 60 \text { miembros, con } \\
\text { una participación balanceada de } \\
\text { hombres y mujeres. El trabajo } \\
\text { diario se organiza a partir de } \\
\text { equipos de dos o tres miembros, } \\
\text { quienes en muchos casos son } \\
\text { parientes, vecinos o coterráneos. }\end{array}$} & \multicolumn{3}{|c|}{$\begin{array}{l}\text { Los grupos tienen } \\
\text { su composición más } \\
\text { reducida, de } 20 \text { a } 25 \\
\text { integrantes, con un } \\
\text { claro predominio de } \\
\text { varones y preferencia } \\
\text { por los trabajadores } \\
\text { experimentados. }\end{array}$} & $\begin{array}{l}\text { Las cuadri- } \\
\text { llas son } \\
\text { "paradas" } \\
\text { durante casi } \\
\text { todo el mes. }\end{array}$ \\
\hline
\end{tabular}

Tabla 1. Expansión y contracción de cuadrillas durante un ciclo productivo de uva de mesa. Elaboración del autor a partir de información recopilada durante el trabajo de campo. 
Dado que las uvas de mesa suelen tener especificaciones definidas en cuanto a su presentación final, el trabajo de todas estas faenas es objeto de un constante control de calidad, tanto en el mismo espacio y momento de realización como en instancias posteriores. Diariamente, los grupos de trabajo reciben de sus mayordomos sesiones de instrucción en donde se comunican los lineamientos de las labores. Tras establecerse lo requerido, el trabajo es objeto de supervisión constante, en primer término, por los propios mayordomos, y de manera adicional, por encargados de las compañías que monitorean de manera general a todos los grupos de trabajo. En las faenas preparatorias se controla que la labor se realice de una forma que garantice un producto de la mejor calidad posible y no se dañen las plantas, mientras que durante la cosecha, cuando ya se obtiene el producto mismo, se busca que este cumpla sus especificaciones de presentación en cuanto a color, tamaño y forma, así como acomodo en el empaque cumpliendo el peso deseado. Los mayordomos llevan un control preciso de lo realizado por cada uno de sus trabajadoresdesde cada surco podado y limpiado hasta cada caja empacada- y el desempeño personal y de los grupos en su conjunto puede ser objeto de evaluaciones precisas. Cuando se detectan fallas, los responsables reciben llamadas de atención, que pueden ir desde observaciones hechas de manera tranquila y amistosa hasta enérgicas descalificaciones, que suelen incluir intimidación y amenazas de despido.

Prácticamente en todas estas tareas el arreglo contractual dominante es el pago por hora, casi siempre con el salario mínimo estatal, complementado en algunos casos por incentivos a la productividad, como bonos por cada cierto número de cajas empacadas o por cantidad de surcos podados. Durante la temporada de cosecha, los miembros de las cuadrillas pueden trabajar regularmente entre 35 y 45 horas por semana, pero durante las tareas complementarias el número de horas suele ser menor y más irregular. El trabajo se ejecuta normalmente durante las mañanas, arrancando poco después del amanecer. A lo largo del ciclo las jornadas suelen tener una duración de entre 6 y 9 horas de trabajo efectivas, con uno o dos descansos de 10 a 15 minutos, y uno de media hora, no pagado, dedicado al lunch.

\section{Administración de la mano de obra: expansión y contracción de cuadrillas}

Extendida en una superficie de más de 20,000 hectáreas, la producción de uva de mesa de la zona está planificada y distribuida regionalmente para conseguir la presencia en el mercado durante el mayor tiempo posible. Por ello, las distintas variedades de fruta y sus ciclos se programan para que su maduración se dé de manera espaciada y no simultáneamente. Resultado de esto, el trabajo a lo largo del ciclo también se planifica para cubrir, en momentos diferentes, todas las tareas en una gran superficie. Ello demanda mecanismos efectivos de organización de la mano de obra, capaz de movilizarla y adaptarla en respuesta a las necesidades variables. Aquí, una estructura de intermediación entre las empresas y los trabajadores, compuesta por contratistas y mayordomos, juega un rol esencial. Como sucede en casi todo el empleo agrícola en California, la fuerza de trabajo de las uvas para mesa es manejada a través de la práctica de sub-contratación. Aun delegando en esta instancia de intermediación la parte administrativa del manejo del trabajo, las compañías productoras buscan que su base de trabajadores sea estable y recurrente 
y cuente con las habilidades necesarias para la realización de las tareas, creándose una relación continua pese a no ser directamente empleadores. Como las posiciones en los grupos no son permanentes, son los intermediarios quienes se encargan de propiciar la estabilidad y recurrencia no obstante la variabilidad estacional, regulando la expansión y contracción del contingente de trabajadores.

Para observar los mecanismos de adaptación de la plantilla de empleados, podemos tomar como ejemplo una cuadrilla en la que se sondearon distintas variables. Para el momento de la cosecha, esta cuadrilla contaba con 60 elementos, agrupados en 30 equipos de dos integrantes. Acorde con una práctica que se encontró de manera recurrente al estudiar otros grupos de trabajo, parte de estos equipos más pequeños se integraban por trabajadores con relaciones personales previas: 11 eran parejas de esposos, 3 de padres-hijos y 1 de primos. Treinta y dos de los miembros eran mujeres y 28 varones. Veinte miembros del grupo, de los cuales 17 eran varones, permanecían con él todo el año, y cada uno de ellos tenía una trayectoria mínima de 7 años en la compañía. Otros 12 miembros integraban el grupo durante 9 meses, arrancando su participación en la primavera y cesando en el invierno, y 16 estaban solo durante la cosecha, transitando al terminar a otros cultivos, especialmente la cosecha de cítricos extendida en las faldas de los límites montañosos del Valle. El mayordomo de la cuadrilla llevaba cuatro años con el control del grupo, habiendo sido antes asistente ("segundo") del mayordomo anterior. Al tomar el mando conservó a buena parte del personal de base original, a quienes sumaba de manera temporal, para la pizca, a un grupo de vecinos del poblado en que residían en California.
Según se apuntó, para julio y durante los siguientes cinco meses, las cuadrillas tienen un tamaño que va de los 50 a los 60 miembros, mientras que entre los meses de diciembre y febrero tienen su tamaño más reducido, de 20 a 25 integrantes. Para constituir un grupo que pueda adaptarse a tales variaciones, los mayordomos, como responsables de las cuadrillas, desarrollan un núcleo base más estable, que se emplea durante todo el ciclo y una red de refuerzos que se integran estacionalmente. Esta diferencia entre una posición permanente y estable y, la participación temporal, se relaciona con el término de "señoría". El concepto no se refiere necesariamente a una disposición oficial y contractual, pues en muchas compañías el reconocimiento de la trayectoria no existe como tal, sino que se usa más bien como una forma coloquial de definir la prioridad que un trabajador desarrolla conforme se mantiene en un grupo, adquiriendo la posibilidad de ser tomado en cuenta de manera preferente para participar en el mayor número de tareas $\mathrm{y}$, por lo mismo, conservarse empleado durante mayor tiempo.

Dentro de este ciclo variable, para conseguir la expansión del número de trabajadores cuando las cuadrillas alcancen su mayor tamaño, el primer medio es la incorporación de miembros recurrentes, ya conocidos, vinculados a los trabajadores del núcleo base. El mayordomo de la cuadrilla que nos sirviera de ejemplo explicaba que para asegurarse de contar con todos los trabajadores necesarios al momento de la cosecha se apoyaba de esta forma en los refuerzos que le proporcionaban los propios miembros permanentes en su cuadrilla: 
“(...) de los que traigo todo el año, casi todos están casados y cuando se viene la cosecha traen a sus esposas, o a un hermano o un primo, y así es con casi todos cada año, y para cuando se acerca la pizca ya sé más o menos con cuántos puedo contar y si voy a necesitar buscarle por otro lado".

Además de esta forma de expansión, otro recurso consiste en agregar a un grupo de conocidos que se integran en conjunto para el período de la cosecha sin tener otra vinculación con la cuadrilla el resto del año. En nuestro ejemplo, el mayordomo incorporaba conjuntamente a un grupo de doce parientes y paisanos, todos ellos migrantes recientes originarios del mismo pueblo en México (Mitlalzingo, Guerrero), a quienes conoció al compartir con algunos de ellos sus horas de descanso en las canchas de basquetbol del poblado en que vivían.

La obtención de la permanencia dentro de un grupo no es automática. Si bien durante el periodo de expansión más grande, la cosecha, pueden incorporarse novatos ajenos al trabajo en los viñedos e incluso carentes de experiencia en el trabajo agrícola, su incorporación a otras tareas de mayor complejidad se dará de manera paulatina, en la medida que demuestren ante sus jefes disciplina y capacidad para realizar su trabajo sin necesidad de supervisión y corrección constante. En este sentido, la permanencia en un grupo de trabajo, que depende menos de un arreglo contractual y más de una relación personal, es resultado de un proceso de aprendizaje técnico en cuanto a una adquisición de destrezas pero también fruto de un posicionamiento en una estructura social determinada. Esto implica construir y ampliar una serie de relaciones personales con diversos empleadores, aprovechando los contactos de familiares, amigos o vecinos que cuentan con trabajo, haciéndose notar ante ellos como un trabajador "deseable", de manera que se consiga paulatinamente mayor integración y cercanía con las opciones de empleo más estable.

Como se vio, alrededor del $35 \%$ de los trabajadores que integran una cuadrilla durante la temporada de trabajo más larga conservan su lugar en el grupo cuando esta termina y cuentan con un empleo casi permanente, con algunos lapsos de desocupación de distinta duración. Si tienen documentación migratoria y laboral en regla, durante esos periodos pueden recurrir al seguro de desempleo. Aproximadamente otra tercera parte encuentra una posición en el grupo por ocho o nueve meses, y el resto es requerido únicamente en el auge de la cosecha. Para mantenerse empleados y obtener un ingreso con el cual garantizar su subsistencia, quienes son detenidos buscan otras oportunidades dentro del mismo empleo agrícola, y una de las opciones mencionada de manera recurrente es la producción de cítricos, fundamentalmente naranja, también de gran importancia en esta zona del Valle. Gracias a un calendario que puede resultar complementario por arrancar su cosecha aproximadamente cuando la de las uvas termina, para extenderse durante los meses siguientes, el tránsito entre cultivos dentro de una misma región se ha convertido en una alternativa preferida sobre el desplazamiento geográfico como medio para mantenerse empleado.

Sin alcanzar la relevancia que tienen los cítricos por su extensión territorial y larga temporada de empleo, la variedad y expansión de la producción agrícola puede brindar a quienes trabajan temporalmente en los viñedos 
opciones de empleo complementario. Por ejemplo, al ser "parados" en sus cuadrillas por el término de la temporada de cosecha, pueden encontrar acomodo en labores como la poda de almendros ${ }^{4}$. Durante los meses de invierno, cuando la demanda de trabajo en la zona muestra una disminución general, algunos pueden encontrar acomodo en la limpieza de campos. Con la llegada de la primavera, quienes no son tomados en cuenta en las primeras expansiones de sus cuadrillas, pueden encontrar trabajo por pequeñas temporadas en cosechas intensivas de corta duración, como las cerezas o las moras azules. Para otros, especialmente si no son experimentados en la realización de distintas tareas y no han desarrollado una red extensa de contactos con posibles empleadores, las opciones de colocación en otros puestos de trabajo son más limitadas, y pueden pasar semanas sin trabajar de manera continua ni percibir ingresos significativos, subsistiendo de los ahorros que hayan conseguido durante sus periodos de ocupación y de los pagos aislados que obtienen cuando logran sumarse con algún mayordomo o productor en labores ocasionales e intermitentes como limpiar un campo, acondicionar instalaciones o apoyar en una inesperada contingencia que demande trabajadores.

\section{Los trabajadores}

Con excepción de los filipinos ya mencionados y de otros más escasos centroamericanos, especialmente guatemaltecos y salvadoreños, el trabajo de los viñedos es realizado fundamentalmente por mano de obra mexicana. Buena parte de esta es originaria de lo que se conoce como "zonas tradicionales" de emigración, en el centro occidente de México, pero en fechas recientes destaca la presencia de migrantes de nuevas regiones, muchos de ellos indígenas provenientes del sur del país ${ }^{5}$. Al momento de la investigación, la participación de estos nuevos migrantes en el trabajo en los viñedos se concentraba en las cosechas, siendo pocos los que contaban con posiciones más estables y regulares abarcando el ciclo completo.

A diferencia de lo que puede observarse en otros trabajos como el corte de verduras, que tienen un claro predominio de jóvenes, con la visita a varias cuadrillas empleadas en la uva de mesa puede apreciarse que el rango de edades de los trabajadores en esta tarea es bastante amplio: igualmente pueden verse trabajadores jóvenes, de 17 o 18 años, que hombres y mujeres que rondan los 60. Algunos informantes mayores comentaron que esto se debe a que tanto la cosecha como los otros trabajos que forman parte del ciclo de las uvas son tareas físicamente menos agotadoras y rápidas, en las que la experiencia les permite ser competitivos. También llamativa en comparación con otros espacios agrícolas es la notoria presencia femenina, que como hemos mencionado se equipara a la de varones en ciertas etapas del ciclo.

La mayoría de los trabajadores que llegaron a Estados Unidos antes de 1986, cuentan con documentación migratoria regular, pues aun habiendo entrado al país de manera indocumentada tuvieron la oportunidad de regularizar su situación aprovechando la implementación de la Immigration Reform and Control Act (IRCA), que fue particularmente favorable para los trabajadores agrícolas ${ }^{6}$. Quienes han migrado después que se diera esa última amnistía y hasta las fechas actuales permanecen mayoritariamente en situación indocumentada, encontrando la facilidad de trabajar en la agricultura con documentación paralegal por el 
menor control implementado en el sector, pero quedando marginados del acceso a una serie de beneficios que se limitan a población con estatus de residencia regular, como el seguro de desempleo, los programas de capacitación o las opciones de vivienda subsidiada para trabajadores agrícolas, sufriendo adicionalmente el estigma de "ilegalidad" que suele emplearse como instrumento disciplinario tanto en el trabajo como en otros contextos.

Los trabajadores de los viñedos viven generalmente en las localidades del Valle de San Joaquín, casi siempre en pequeñas ciudades (de entre 5,000 y 20,000 habitantes). Muchos de los que llevan más tiempo viviendo en Estados Unidos, relatan que al momento de sus primeros viajes pasaron por alguno de los espacios habitacionales exclusivos para trabajadores agrícolas que existían esparcidos entre los campos y eran proporcionados por los propios empleadores como parte del acuerdo contractual. Conocidos coloquialmente como campamentos -lo que señalaba su carácter excluyente-, estos espacios han ido desapareciendo, tanto por la presión que comenzó a generarse para mejorar las precarias condiciones en las que solían operar como por el deseo de los migrantes por buscar acomodos más propicios, especialmente cuando se trataba de familias ${ }^{7}$. Ello los condujo a incrementar su presencia en las diversas localidades del Valle, expandiéndose en muchos casos de habitar exclusivamente en secciones segregadas (generalmente conocidos como barrios o colonias mexicanos) a convertirse en mayorías demográficas en varios poblados (Palerm, 2010).

A diferencia de lo que se registra en otros cultivos, como la lechuga, donde cuadrillas enteras de lechugueros son llevados a los campos en camiones del empleador, los trabajadores de la uva suelen llegar a los campos por sus propios medios, organizándose para viajar en grupo si alguno de sus parientes o amigos cuenta con carro, a quien pueden pagar una pequeña cooperación por el transporte. Tampoco está extendido el servicio de los "raiteros" particulares que se dedican exclusivamente a movilizar personal, pero sí es una práctica común que los mayordomos presten este servicio a sus trabajadores que no cuentan con forma de desplazarse, en especial cuando se trata de personas de reciente llegada y nueva incorporación al grupo. En estos casos, el servicio sí tiene un costo preestablecido, y algunos mayordomos convierten esto en un ingreso adicional a su salario y en una forma de control de personal. Debido a esta práctica de dejar la movilidad en manos de los mismos trabajadores, conseguir un vehículo propio se convierte en una prioridad para los migrantes que llegan a trabajar a la zona.

\section{El asentamiento en California}

Tanto los nuevos migrantes que llegan a la zona en fechas recientes como quienes llevan ya una experiencia migratoria de varias décadas, suelen mencionar que su inserción en el empleo agrícola en California forma o formaba parte de un plan temporal, con miras a adquirir ingresos destinados a emplearse en México tras un proyectado regreso. Con los planes de capitalizarse para construir una casa, financiar su propia producción agrícola, establecer un negocio, continuar con estudios escolares o simplemente obtener un ingreso en un momento de situación económica desfavorable, la opción del Norte resulta atractiva. Sin embargo, para muchos de ellos este proyecto se extiende y el regreso a México se demora o 
incluso se pospone de manera indefinida. Las dificultades encontradas para cumplir con sus proyectos, pero también nuevas oportunidades que no se tomaran en consideración al elaborar los planes pueden influir en estos cambios.

Por las características que mencionamos en cuanto a sus requerimientos de trabajo humano, la expansión de la producción de uvas para mesa ha contribuido al aumento de la oferta de empleo agrícola, tanto temporal como de larga duración, y por ende, incentivado la presencia y asentamiento de población mexicana en las zonas en que se desarrolla. Su crecimiento forma parte de un proceso más amplio de "intensificación" de la industria agrícola del estado, motivado por el crecimiento en importancia de cultivos como las fresas, naranjas, brócoli o lechuga, que, de manera semejante a lo que hemos visto aquí, dependen en su producción de la participación de elevadas masas de trabajadores (Palerm, 1991; Khan, Martin \& Hardiman, 2004).

Con temporadas más largas de empleo local vinculado a un mismo patrón, algunos trabajadores de la uva comenzaron a abandonar el seguimiento de los recorridos de trabajo que se desplazaban por circuitos extensos, a veces abarcando más de un estado, para permanecer en cambio por más tiempo en un mismo punto durante sus estancias en Estados Unidos. Gracias a la apertura a la presencia femenina dentro de las cuadrillas de trabajo (que no se repite en todos los ámbitos del empleo agrícola), algunos trabajadores incorporan a sus esposas o hermanas en el flujo migratorio, buscando crear un ingreso más alto, favoreciendo una transición de la migración masculina y circular a esquemas con mayor presencia de familias enteras. En la medida en que estas familias incorporan a sus descendientes - muchos de los cuales ya son ciudadanos nativos estadounidenses- al sistema escolar y en general al nuevo medio al que se han mudado, construyen nuevas expectativas, contemplando que sus hijos por ser solventes en el uso del inglés, cuenten con educación formal y no tengan la limitación del estatus de indocumentado, podrán aspirar a mejores empleos y posibilidades profesionales más satisfactorias que aquellas que podrían encontrar en México, con lo que la permanencia en Estados Unidos se vuelve más atractiva.

Además, durante las primeras fases de esta intensificación, un proceso de mejora de las condiciones laborales, resultado de las luchas encabezadas por el sindicato United Farm Workers bajo el liderazgo de Cesar Chávez y Dolores Huerta (Sosnick 1978: 349-350; Martin, 2004) generó también mayores alicientes para la estabilización de los trabajadores. Teniendo la industria de la uva de la mesa como uno de sus bastiones más fuertes, la movilización propulsada por el UFW consiguió elevar los salarios y la adquisición de ciertas prestaciones (como seguro médico) en sus contratos con algunas compañías. Además consolidó una base de negociación legislativa que logró extender al empleo agrícola algunas prestaciones laborales que ya operaban en otros sectores de la economía desde hacía varias décadas, como el propio reconocimiento legal y formal de la representación sindical, que se reglamentó en 1975 con la disposición del Agriculture Labor Relations Act, y la implementación del seguro de desempleo, que se otorgó en 1978 y contribuyó a la extensión de la estancia en Estados Unidos al suministrar un ingreso para enfrentar los periodos de desocupación ${ }^{8}$. La regularización masiva de la situación migratoria resultante de IRCA, al incrementar el número de posibles beneficiarios, potenció el efecto de tales medidas. 
Además de estos alicientes, otros factores, como el endurecimiento de los controles migratorios en las zonas fronterizas, que se agudizan desde mediados de los 90 , provocan que quienes migran opten por temporadas de permanencia más larga. Si durante la década de 1970 la lasitud del control fronterizo permitía entrar con relativa facilidad de manera indocumentada, favoreciendo la realización de viajes continuos al término de las temporadas de empleo, las mayores restricciones que operan actualmente dificultan y encarecen considerablemente el cruce, disuadiendo la circularidad. Dado que el costo económico que tiene el cruce es pagado generalmente por los propios migrantes -lo que suele implicar que contraigan una deuda para incorporarse al empleo en Estados Unidos-, la opción de visitar México con regularidad se vuelve poco rentable, siendo preferible mantenerse en las localidades a las que han llegado buscando trabajo, aún durante los lapsos de desempleo, pues cada nueva entrada los obliga a dedicar parte de sus ingresos al pago de los "coyotes" que los llevan hasta su lugar de destino ${ }^{9}$. Este endeudamiento suele influir también en la dilación del original proyecto de retorno a México, pues la liquidación de la deuda puede tardar más de lo pensado, en especial para aquellos que no encuentran empleo de manera regular a su llegada. En conjunto, los gastos de manutención y el pago de la deuda agotan la mayoría de los ingresos durante los primeros meses, y es hasta que la deuda se ha saldado que se está en condiciones de hacer ahorros significativos.

Todo lo anterior ha contribuido a un proceso en el que parte de la población de trabajadores agrícolas, otrora móvil y revolvente, se ha "sedentarizado" (Palerm, 1999), estabilizándose en distintas localidades del medio rural californiano, de manera más notoria en aquellas regiones en las que la agricultura se reestructuró más radicalmente hacia la producción de mercancías intensivas en uso de fuerza de trabajo. En varios sentidos, en este amplio proceso se ha transformado el arreglo social de la producción agrícola californiana, aunque bajo un esquema que carga inequitativamente muchos de los costos a los propios trabajadores. Adicionalmente, la política migratoria genera no solo condiciones de exclusión que imposibilitan a los trabajadores disfrutar plenamente de los beneficios que les corresponderían como miembros productivos de la economía, sino oportunidades perversas para sistemas de abuso, como los artificiales costos que los coyotes y contratistas pueden imponer a la participación en el trabajo.

La nueva dinámica productiva está llena de tensiones y contradicciones. Gracias a un sistema social que incorpora redes de solidaridad basadas en parentesco, amistad y paisanaje, la reserva de mano de obra flexible requerida en las necesidades variables de la producción contemporánea puede acercase y estabilizarse, pero también disciplinarse y controlarse disuadiendo los reclamos y la movilización sindical. Con las extensiones en los tiempos de trabajo y las ampliaciones de los calendarios se crean mayores opciones de empleo, pero este sigue sin ser equivalente a una posición permanente, además de ser remunerado en los niveles más bajos. Imperan los bajos salarios (alrededor del salario mínimo), con los que inclusive un trabajador que participara en el ciclo completo de los viñedos no conseguiría lo suficiente para superar la línea de la pobreza para una familia de cuatro integrantes si el suyo fuera el único ingreso ${ }^{10}$. Quienes trabajan con menor regularidad sufren aún mayores carencias. Por ello, la proporción 
de trabajadores que pese a estar empleados quedan por debajo de la línea de pobreza es más alta en el trabajo agrícola que en cualquier otra actividad (Bugarin \& López, 1998: 51). Finalmente, aunque el asentamiento propicia que la fuerza de trabajo agrícola participe del mercado local de manera más intensa en rubros básicos como los gastos de subsistencia, vivienda, transporte y servicios, esto sucede desde posiciones desventajosas que pueden conducir a condiciones desfavorables. Quizá el ejemplo más elocuente lo represente el acceso a vivienda, que debido a los bajos ingresos en no pocas ocasiones se subsana en situaciones de sobreocupación, uso de espacios no habitacionales (como cocheras, bodegas o pórticos) o renta de unidades deterioradas y con servicios precarios (Villarejo, 2013). Con posibilidades muy reducidas para conseguir los ingresos suficientes para vivir de acuerdo a los estándares estadounidenses, es gracias a la aceptación de condiciones menos favorables y al respaldo que brinda una comunidad cada vez más rica en vida social que se genera el contexto para la funcionalidad de una clase trabajadora local activa en la agricultura.

\section{Conclusiones: ¿un nuevo tipo de trabajador agrícola?}

Algunos observadores han caracterizado el uso masivo de mano de obra asalariada en la agricultura californiana como resultado de la disponibilidad de una preexistente y ajena sobredotación de trabajadores (Fuller, 1939; Martin, 2002). Sin embargo, también se ha señalado que este énfasis en el lado de la oferta de trabajadores ha restado atención tanto a la creciente necesidad de trabajadores de una industria que se reestructura (Palerm, 1991) como a los mecanismos que activamente han sido impulsados por los empleadores para reclutar y mantener los contingentes de brazos demandados (Krissman, 2000). Con la intención de contribuir al entendimiento de estas dinámicas desde el énfasis en los espacios de trabajo, aquí nos hemos interesado por los procesos de proletarización atendiendo menos las condiciones de desposesión en las regiones campesinas de México (los lugares de origen de los migrantes), y más las situaciones de inserción y participación en los contextos locales de destino: la agroindustria en el Valle de San Joaquín. Esta atención a las condiciones particulares tiene en mente la observación de Harvey de que "no importa qué tan universal sea el proceso de proletarización, el resultado no es la creación de un proletariado homogéneo" (Harvey 2003: 147). Así, buscamos aportar al entendimiento del tipo de proletario que surge con la sedentarización de los trabajadores agrícolas migrantes.

Como punto de partida de esta reflexión final, podemos recuperar las formulaciones propuestas por S. Mintz en sus estudios de las plantaciones azucareras caribeñas. Mintz apuntó que el proletariado rural se caracteriza por carecer de tierra, ser empleado por corporaciones, ser remunerado con un salario y por comprar en tiendas los insumos para su subsistencia (Mintz, 1974). Con lo expuesto aquí, podemos ver que todas estas características están presentes en el caso que nos ocupa: los trabajadores mexicanos que se emplean en la industria de la uva para mesa en el Valle de San Joaquín se incorporan a un sector dominado por empresas medianas o grandes y no por pequeños productores; dependen de un salario para su subsistencia y, desde que no reciben transporte, vivienda ni alimentación por parte de sus empleadores, la garantizan adquiriendo por su cuenta sus satisfactores. Así, en primera instancia su carácter de proletarios 
parece estar claramente definido. Sin embargo, recuperando nuestro interés por la formación de clase, podemos cuestionar el alcance de estas condiciones para la conformación de una clase trabajadora semejante a la de otras esferas de la economía capitalista. Al reflexionar sobre la proletarización del trabajo rural, el propio Mintz apuntó que se trata de un proceso que puede describirse "en términos de cuatro aspectos principales: racionalización de la producción; consolidación de membresía de clase; el crecimiento de una conciencia aparejada a tal membresía; y la individualización de la clase trabajadora" (Mintz, 1974: 319). Estos cuatro elementos han tenido un desarrollo notorio en el caso de la agricultura californiana, pero no de una forma lineal y uniforme, sino con tensiones y contratendencias. Así, la constante inversión tecnológica y el incremento de la organización de la producción bajo estilos empresariales modernos no solo generan extensiones de tiempos de trabajo que fomentan la estabilización de los trabajadores, sino también conducen a un espacio productivo racionalizado en donde el control del proceso de trabajo queda en manos de la administración capitalista, bajo esquemas de alienación semejantes a los de otras industrias. Sin embargo, podemos señalar una contratendencia resultante de la destacada importancia de las relaciones interpersonales y los arreglos basados en normas culturales que forman parte de los mecanismos usados tanto por contratistas y mayordomos para el manejo de la mano de obra, como por los propios migrantes para sortear las condiciones desfavorables en el entorno económico estadounidense. Igualmente, el auge de la movilización sindical que se experimentó durante algunos años puede entenderse como una manifestación de consolidación de clase, pero su paulatino debilitamiento podría verse como un signo de una tendencia contraria. En fechas recientes, una movilización social más abarcadora, que enfatiza parte de su lucha en la condición migratoria y en las definiciones de ciudadanía -aunque destacando de manera fundamental la condición de clase trabajadora de la población migrante- pareciera apuntar a nuevas formas de movilización social. En todos estos puntos recién mencionados, el carácter inmigrante de esta fuerza de trabajo emerge como un factor crítico y distintivo. ¿Qué representa la condición forastera de estos trabajadores para su proletarización?

Históricamente, la participación de los migrantes mexicanos en la agricultura capitalista de California ha sido como trabajadores que se incorporan vendiendo su fuerza de trabajo y convirtiéndose en asalariados, de lo que podemos desprender que se trata de una forma de proletariado. Sin embargo, al menos mientras imperaron patrones de migración circular, ese proletariado no permanecía vinculado de manera estable a la producción capitalista, que no lo requería permanentemente. En muchos casos contaba con el acceso a medios de producción propios en el ámbito rural mexicano, de los que podía obtener ingresos que le permitían subsistir mientras no devengaba un salario. Esta modalidad de participación en la agricultura capitalista posibilitaba lo que M. Burawoy definió como "la externalización, a una economía y/o estado alterno, de ciertos costos de la renovación de la fuerza de trabajo -costos normalmente asumidos por el empleador y/o el estado de empleo" (Burawoy, 1976: 1050). Así, éste era un tipo especial de proletario que podemos identificar con lo que C. Meillassoux definiera como proletariado-campesino, un tipo de trabajador que "solo recibe del capitalismo los medios para la recons- 
titución inmediata de su fuerza de trabajo, pero no para su mantenimiento y su reproducción, medios que él se procura en el marco de la economía doméstica" (Meillassoux, 1979: 189). Sin embargo, en las dinámicas productivas y migratorias que describimos arriba, que fomentan permanencias más prolongadas en Estados Unidos, el recurso a los medios de la economía doméstica y campesina en México se ve parcialmente imposibilitado. ¿Estamos entonces ante proletarios plenos?

Junto a su definición de proletariado-campesino, Meillassoux propuso la categoría de proletariado integrado para caracterizar a aquellos trabajadores que participan en el sistema capitalista y reciben un salario directo de su empleador para cubrir su reconstitución y un salario indirecto consistente en una serie de beneficios complementarios para garantizar su reproducción, ya sea en la forma de satisfacción de derechos sociales en un sistema socializado por el Estado de bienestar (modalidad más común en el capitalismo occidental europeo) o en una combinación de esto con arreglos contractuales en el sistema de mercado (situación perseguida en los Estados Unidos de América). Recuperando esto como guía, podemos sugerir que la reestructuración productiva y la estabilización de fuerza de trabajo propician la existencia de lo que podemos entender como un proletariado con integración fragmentaria y diferencial, con diversos factores que impiden que la fuerza de trabajo agrícola se equipare del todo a las clases trabajadoras de otros sectores de la economía estadounidense.

Primeramente, las variaciones impuestas por los ciclos agrícolas, aunque cada vez más matizadas, siguen imponiendo obstáculos para una participación estable en el empleo y, por ende, para la obtención regular de un salario directo. Además, las posibilidades de conseguir los estándares locales de otros asalariados estadounidenses (integración) están limitadas por el carácter excepcional tanto legal como simbólico del empleo agrícola: imperan una muy reducida extensión de beneficios laborales y una sistemática infravaloración del trabajo en el campo. Esto opera en una forma diferencial para diversos actores pues tanto la propia participación en el empleo como el acceso a los beneficios se organizan obedeciendo a clasificaciones y distinciones simbólicas de situación y trayectoria migratoria, género, edad y etnicidad. Todo ello está, además, cruzado por un orden transnacional en el que el arreglo jurídico-simbólico emanado de las fronteras posiciona de maneras distintivas a los mexicanos que penetran a la economía estadounidense, imponiendo categorías como "ilegalidad", que posibilitan los mecanismos de exclusión y sobreexplotación (Kearney, 2006).

La aproximación al proceso de proletarización expuesto aquí deja diversas interrogantes abiertas para nuevas investigaciones. Es necesario explorar con mayor profundidad los mecanismos de reproducción de la mano de obra en un contexto en el que se reduce la externalización hacia economías alternas pero no se adquieren los salarios indirectos dentro de la economía estadounidense. Igualmente, la presencia de una generación de hijos de inmigrantes, formados al menos parcialmente dentro de los ámbitos de la sociedad receptora, nos invitan a cuestionarnos si ellos serán eventualmente los reemplazos generacionales en los campos (lo que no parece estar sucediendo) o si conseguirán evitar, como es el deseo de sus padres, el empleo agrícola. Y si esto sucede, ¿continuará una dependencia de arribos desde el exterior para la renovación del trabajo? 
¿Cuáles serán, entonces, las trayectorias ocupacionales de las segundas generaciones? Por último, ejercicios comparados con otros casos de re-intensificación agrícola se antojan necesarios para establecer puntos de confluencia y divergencia de las dinámicas laborales. Si, como apunta A. Pedreño (2011), la "condición inmigrante" es la característica definitoria del trabajo en las agriculturas intensivas globales, las peculiaridades de los contextos locales y de las especificidades de distintas experiencias migratorias se vuelven temas relevantes para comprender las variaciones de una tendencia mundial.

En Estados Unidos, la contribución del trabajo agrícola asalariado es a menudo negada o minimizada, y su identificación con los escaños más bajos de las jerarquías ocupacionales conduce a la infravaloración de las propias personas que realizan esta labor. Como apunta P. Benson (2008), al escatimársele su valor, las imágenes negativas que se asocian al trabajo agrícola respaldan percepciones que normalizan condiciones de exclusión, explotación y desatención estatal. En este escrito, hemos buscado entender a los migrantes trabajadores agrícolas mexicanos como participantes activos y hoy todavía necesarios en una valiosa y compleja industria. Quizá en un futuro la agricultura se mecanizará, o se desplazará por presiones ecológicas o cambiará por una nueva reestructuración productiva, pero consideramos que el aporte que hasta ahora hacen los trabajadores debe ser reconocido, tanto con la mejora de los beneficios laborales como con una solución de la situación migratoria que solo carga a sus espaldas mayores desventajas.- 


\section{Notas}

${ }^{1}$ Trabajadores traídos de Asia (China, Japón, India y Filipinas) en sucesivas oleadas entre finales del siglo XIX y principios del XX; desplazados internos empujados por la Gran Depresión de 1929; y migrantes mexicanos en distintos momentos, forman parte de la historia de una numerosa fuerza de trabajo llegada desde el exterior.

2 De acuerdo a los estudios de especialistas en agronomía (Mammer \& Wilkie, 1990), la producción de uvas de mesa puede alcanzar requerimientos de fuerza de trabajo dos veces más altos que cultivos como la lechuga o nueve veces mayores a los del algodón.

3 Los viñedos del Valle de San Joaquín son uno de los pocos espacios de California en los que los trabajadores agrícolas mexicanos comparten las tareas del campo con personas de otro origen étnico, principalmente con los migrantes filipinos que hace algunas décadas fueran más numerosos en esta labor y en la que aún permanecen algunos, si bien en número cada vez más reducido y, al parecer, sin renovarse con nuevas generaciones que lleguen a incorporarse al empleo agrícola en la zona.

${ }^{4}$ Las almendras son un cultivo mecanizado con bajos requerimientos de mano de obra a lo largo del año, por lo que pese a extenderse en una superficie mayor, no genera una base de trabajadores semejante a la de las uvas o las naranjas, demandando la presencia de jornaleros por periodos cortos y aislados, como las podas posteriores a las cosechas.

5 Durand \& Massey (2003: 159) sugirieron que la agricultura californiana podría experimentar un proceso de "indigenización" para satisfacer la creciente demanda de mano de obra, al ser la población indígena la siguiente reserva de trabajadores disponibles en el medio rural mexicano. Es probable que este proceso se esté generando, aunque lentamente: de acuerdo a un estimado, la proporción de indígenas mexicanos en el conjunto de los trabajadores agrícolas de California pasó del 5 al 15\% entre 1997 y 2009 (CRB, 2013).

${ }_{6}$ Aunque la idea original de IRCA (también conocida como Ley Simpson-Rodino) era establecer distintos controles para detener el flujo de migración indocumentada, especialmente a través de sancionar a empleadores que conscientemente contrataran personal indocumentado y del fortalecimiento de la vigilancia fronteriza, la reforma incluyó un programa de "amnistía" para migrantes que hubieran entrado al país previamente, con el que aproximadamente tres millones de migrantes (dos terceras partes de los cuales eran mexicanos) consiguieron regularizar su situación. El empleo agrícola contó con tres programas especiales, a través de los cuales era más sencillo cubrir los requisitos para la regularización si se demostraba haber sido trabajador del campo. Esto favoreció no solamente la permanencia de quienes en ese momento constituían la fuerza de trabajo agrícola, sino también la incorporación de sus familiares que residían en México y encontraron facilidades para trasladarse a EU y regularizar rápidamente su situación (Phillips \& Massey, 1999: 233; también Martin, 2004 \& Palerm, 1999).

7 Se estima que de más de 5,000 campamentos existentes en 1964 quedan en operación menos de una quinta parte (Villarejo, 2013: 1).

${ }^{8}$ Pese a sus triunfos significativos, la capacidad de intervención de UFW en el mercado de trabajo agrícola comenzó a disminuir paulatinamente hacia finales de los 70 y comienzos de los 80 . En las últimas décadas su presencia como representación oficial de los trabajadores en la industria de la uva prácticamente ha desaparecido.

9 Se conoce coloquialmente como "coyotes" o "polleros" a quienes conducen a los migrantes en el cruce subrepticio de la frontera, a cambio de un pago. Para el momento en que se realizó la investigación, durante 2006-2007, los costos de estos servicios, según los propios informantes, iban de 1,500 a 2,500 dólares por persona, cruzando por el desierto entre Sonora y Arizona. La variación dependía del punto final de destino. En muchos casos, se acuerda pagarle esta "tarifa" al pollero durante los siguientes meses después de llegar a EUA y conseguir trabajo. Otras veces el apoyo para el pago proviene de ahorros, deudas contraídas en México o con algún pariente o conocido ya establecido en el lugar de destino, con quienes igualmente existe el acuerdo de saldar la deuda conforme se empiece a obtener un ingreso.

${ }^{10}$ De acuerdo a los lineamientos federales (USCB, 2015), la línea de pobreza a mediados de la investigación (2006) era de 20,614 dólares para una familia de cuatro. Mientras, el salario mínimo vigente entonces en California era de 6.75 dólares por hora. El ingreso de un trabajador regular (incluyendo bonos y percepciones de seguro de desempleo), alcanzaría apenas para aproximarse a un 65-70\% de esa línea. 


\section{Referencias bibliográficas}

Benson, P. (2008). "El Campo. Faciality and Structural Violence in Farm Labor Camps". Cultural Anthropology. Vol. 23, № 4.

Bonnano, A., Busch, L., Friedland, W., Gouveia, L. \& Mingione, E. (1994). From Columbus to ConAgra. The Globalization of Agriculture and Food. Kansas: University Press of Kansas.

Boriss, H., Brunke H. \& Kreith, M. (2006). Commodity profile: Table grapes. Agricultural Issues Center, University of California. Disponible en: http://aic.ucdavis.edu/profiles/ GrapesFreshMarket-2006.pdf Fecha de consulta: 12 de julio de 2015.

Bugarin, A. \& Lopez, E. (1998). "Farmworkers in California. Sacramento: California Research Bureau". Disponible en: https:// www.library.ca.gov/crb/98/07/98007a.pdf Fecha de consulta: 12 de julio de 2015.

Burawoy, M. (1976). "The Functions and Reproduction of Migrant Labor: Comparative Material from Southern Africa and the United States". American Journal of Sociology, Vol. 85, № 5 .

CDFA, California Department of Food and Agriculture (s/f). California County Agricultural Commissioner's Reports 2012. Sacramento: CDFA. Disponible en: http://www.nass.usda.gov/ Statistics_by_State/California/Publications/AgComm/201212cactb00. pdf Fecha de consulta: 12 de julio de 2015.

CRB, California Research Bureau (2013). Farmworkers in California: A Brief Introduction. CRB. Disponible en: http://www.library. ca.gov/crb/13/s-13-017.pdf Fecha de consulta: 12 de julio de 2015.

Durand, J. \& Massey, D. (2003). Clandestinos. Migración México-Estados Unidos en los albores del siglo XXI. México: Miguel Ángel Porrúa, Universidad de Zacatecas.

Feder, E. (1977). "Campesinistas y descampesinistas. Tres versiones divergentes (no incompatibles) sobre la destrucción del campesinado". Comercio Exterior, Vol. 27, № 12.

Fisher, L. (1953). The Harvest Labor Market in California, Harvard: Harvard University Press.

Fuller, V. (1939). The Supply of Agricultural Labor as a Factor in the Evolution of Farm Organization in California. (Disertación Doctoral).Universidad de California, Berkeley.

(1960). "The Economics of Migrant Labor". Social Order, Vol. 10, № 1 .

__ (1974). "Agricultura VI: Trabajo". En Sills, D. Enciclopedia internacional de las ciencias sociales, Vol. 1. Bilbao: Aguilar.

Guerin-Gonzalez, C. (1994). Mexican Workers and American Dream. Immigration, Repatriation and California Farm Labor, 19001939. Brunswick: Rutgers University Press.

Guthrie, J., Lin, B., Reed, J. \& Stewart, H. (2005), "Understanding Economic and Behavioral Influences on Fruit and Vegetable Choices". Amber Waves, № 2. Disponible en: http:// ageconsearch.umn.edu/handle/128235 Fecha de consulta: 12 de julio de 2015.

Harvey, D. (2003). The New Imperialism. Oxford y Nueva York: Oxford University Press.
Hernández, M. (2010). Estabilización de trabajadores agrícolas migrantes: ¿base para su plena proletarización? (Tesis doctoral). CIESAS, México.

Kautsky, K. (1974). La cuestión agraria. México: Siglo XXI.

KCDA, Kern County Department of Agriculture (2015). Kern County Crop Reports. Disponible en: http://www.kernag.com/caap/ crop-reports/crop-reports.asp Fecha de consulta: 12 de julio de 2015.

Kearney, M. (2006) "El poder clasificador y filtrador de las fronteras". En Besserer, F. \& Kearney, M. (eds.). San Juan Mixtepec. Una comunidad transnacional ante el poder clasificador y filtrador de las fronteras. México: Casa Juan Pablos, Fundación Rockefeller, Universidad de California, Riverside, UAM-Iztapalapa.

Khan, A., Martin, P. \& Hardiman, P. (2004). "Expanded Production of Labor-intensive Crops Increases Agricultural Employment". California Agriculture, Vol. 58, № 1.

Krissman, F. (2000). "Immigrant Labor Recruitment: U.S. Agribusiness and Undocumented Migration from Mexico". En Foner, N., Rumbaut, R. \& Gold, D. Immigration Research for a New Century. Nueva York: Russell Sage Foundation.

(1996). California Agribusiness and Mexican Farmworkers (1942-1992): A Bi-national System of Production/reproduction. (Disertación doctoral). Departamento de Antropología, Universidad de California, Santa Bárbara, USA.

Mammer, J. \& Wilkie, A. (1990). Seasonal Labor in California Agriculture: Labor Inputs for California Crops. California Employment Development Department.

Martin, P. (2002). "Mexican Workers and U.S. Agriculture: The Revolving Door". International Migration Review, Vol. 36, № 4. (2003). Promise Unfulfilled Unions, Immigration and Farm Workers. Cornell University Press.

Marx, K. (1975). El Capital. Tomo 1, Vol. 3. Libro primero. El proceso de producción del capital. México: Siglo XXI.

McWilliams, C. (1969 [1939]). Factories in the Field. The Story of Migratory Farm Labor in California. Hamden: Archon Books.

Meillassoux, C. (1979). Mujeres, graneros y capitales, México: Siglo XXI.

Mintz, S. (1974). "The Rural Proletariat and the Problem of Rural Proletarian Consciousness". The Journal of Peasant Studies, Vol. 1, № 3.

Palerm, A. (1998). Antropología y marxismo (2 $2^{\underline{a}}$ edición). México: CIESAS.

Palerm, J. (1991). Farm Labor Needs and Farm Workers in California 1970 to 1989. Sacramento: Employment Development Department.

(1998). "The Expansion of California Agriculture and the Rise of Peasant-Workers Communities". En Jonas, S. \& Thomas, S. (coords.). Immigration: A Civil Rights Issue for the Americas. Delaware: Scholarly Resources.

(1999). "Las nuevas comunidades mexicanas en los espacios 
rurales de Estado Unidos: a propósito de una reflexión acerca del quehacer antropológico". AREAS, № 19.

(2010). "De colonias a comunidades. La evolución de los asentamientos rurales mexicanos en California Rural". En Lara, S. (coord.). Migraciones de trabajo y movilidad territorial. México: $\mathrm{H}$. Cámara de Diputados, CONACYT, Miguel Ángel Porrúa.

Pedreño, A. (2011). "La condición inmigrante del trabajo en las agriculturas globalizadas". En Lara, S. (coord.). Los "encadenamientos migratorios" en espacios de agricultura intensiva. México: El Colegio Mexiquense, Miguel Ángel Porrúa.

Phillips, J. \& Massey, D. (1999). "The New Labor Market: Immigrants and Wages after IRCA". Demography, Vol. 36, № 2.

Sosnick, S. (1978). Hired Hands. Seasonal Farm Workers in the United States. Santa Barbara: McNally \& Loftin, West.
USCD, United States Census Bureau. (2015). Poverty Thresholds by Size of Family and Number of Children. Disponible en: http://www.census.gov/hhes/www/poverty/data/threshld/index. html Fecha de consulta: 12 de julio de 2015.

Villarejo, D. (2013). "California's Hired Farm Workers Move to the Cities: The Outsourcing of Responsibility for Farm Labor Housing". Ponencia presentada en California Rural Legal Assistance Priorities Conference, Asilomar, California. Versión disponible en: http://www. crla.org/sites/all/files/u6/2014/rju0214/VillarejoFrmLbrHsngHlth_ CRLA_012414.pdf Fecha de consulta: 12 de julio de 2015.

Wood, M., McGinnis, L. \& Core, J. (2006). "Grapes! Our Never-ending Crush”. Agricultural Research Magazine, Vol. 54, № 4. 